\title{
LDG METHOD WITH P-ADAPTIVITY APPLIED TO LES OF BLADE-VORTEX INTERACTION
}

\author{
ANDREA P.C. BRESCIANI ${ }^{1}$ AND ANTONELLA ABB $\grave{A}^{2}$ \\ Politecnico di Milano, Dipartimento di Scienze e Tecnologie Aerospaziali \\ Via La Masa, 23 - 20156 Milano (ITALY) \\ ${ }^{1}$ andreapio.bresciani@mail.polimi.it \\ ${ }^{2}$ antonella.abba@polimi.it
}

Key words: Blade-vortex interaction, Large Eddy Simulation, Discontinuous Galerkin, p-adaptivity

\begin{abstract}
In the present work the Local Discontinuous Galerkin (LDG) method with polynomial adaptivity is applied to the Large Eddy Simulation (LES) of the parallel blade-vortex interaction (BVI).

The BVI phenomenon occurs on helicopter and drone rotors in manoeuvring conditions and it produces impulsive changes in the pressure distributions, vibrations and noise. To deeply understand the mechanism of load generation related to the pressure field and three dimensional perturbations growth, to focus on the interaction between the vortex and the three dimensional structures in boundary layer and wake, accurate 3D unsteady numerical simulations of turbulent flows are necessary. For this reason, it is very important the use of a numerical code based on high order schemes such as LDG. Moreover, in the LDG approach, the numerical resolution can be varied on each element and in time, adapting to the requirement of the simulated flow and saving a large amount of computing resources. In the used numerical code the criterion for variation of the polynomial order is based on a refinement indicator especially suited for LES and based on the structure function. The local polynomial representation directly provides a means to separate large from small scale modes, thus providing the starting point for the definition of the subgrid scale models. In the present simulations, the subgrid scales contribution is represented with a sophisticated dynamic anisotropic subgrid model, suitable and well tested for wall resolved LES and complex separated turbulent flows. The BVI is simulated highlighting the effect of the vortex on the pressure distributions, on the boundary layer separation and on the resulting forces.
\end{abstract}

\section{INTRODUCTION}

The interaction of vortices with solid bodies is a complex fluid dynamic phenomenon of common occurrence in a lot of engineering problems such as rotorcraft flows, heat exchangers, bridge pilings, offshore structures and flows around buildings. This interaction usually causes negative effects such as noise, unsteady aerodynamic loads and structural vibration. Both experimental and numerical approaches have been used to better understand the physics behind this phenomenon and to predict the most critical conditions. Particularly interesting from the aeronautic point of view is the BVI in the helicopter rotor where the tip vortex released by a blade interacts with one of the following blades. It is particularly challenging since it usually occurs during slowly descending flight when the rotor wake remains in the proximity of the rotor disk, causing a highly unsteady and three dimensional flow field. The BVI is a relevant problem 
also in the design of Micro Aerial Vehicle (MAV) where a vortex which can be generated either by the flapping motion or by a previous blade (tip vortex) interacts with the low Reynolds flow around the airfoil. However, the BVI is usually classified in three different categories depending [19, 27] on the relative orientation between the vortex axis and the blade span: parallel BVI occurring when the vortex and the blade axis are parallel; perpendicular and orthogonal BVI when the vortex and the blade axis are perpendicular and the vortex axis is perpendicular to or aligned with the stream-wise direction respectively. It is particularly challenging to investigate experimentally the parallel BVI since it is not trivial to generate a single vortex. In a wind tunnel, the vortex generator is usually an airfoil collocated upstream to the target airfoil. The upstream airfoil is moved with a pitch or plunge motion to generate two vortices detaching from the leading edge (LE) and the trailing edge (TE) respectively $[27,18,17]$. Since in an experimental facility to study parallel BVI a LEV and a TEV are always present, the numerical approach is a powerful method to investigate the interaction between an airfoil and a single vortex. The parallel blade-vortex interaction has been studied with a RANS approach, coupled with a vorticity confinement method [16] to prevent an excessive diffusion of the advected vortex due to a poor resolution of the grid. Chimera methods or overlapped grids approaches have been also used in the same framework to predict BVI noise [22]. A hybrid RANS/LES method to study the parallel BVI has been applied by [11], however the first fully LES of a parallel blade-vortex interaction has been made by [14], where a simplified aeroelastic model has been presented. In the present work, the LES approach has been used to investigate the parallel interaction between a vortex and the airfoil. The numerical code is based on the Discontinuous Galerkin finite element method characterised by high order accuracy and very good scalability properties in High Performance Computing facilities. The use of high order numerical method associated with an LES approach not only guarantees a better accuracy of the solution but, furthermore, indoes not require any
hoc technique to prevent the over-diffusion of the vortex. Furthermore the application of the dynarnic
P-adaptive method devgloped by $[23,4]$ allows a consistent paving of computational resources without
losing accuracy.
In the present work, the parallel interaction between a vortex and an dirfoil will be investigated. The used physical and numerical model and the polynomial adaptivity indicator are first presented in Section 2. being the statistically steady state reference condition for the BVI simulation presented in Section 3.2. Then a synthesis of the results and some conclusions are drawn out in Section 4.

\section{NUMERICAL CODE}

\subsection{The physical model}

The considered numerical code solves the Navier-Stokes equations, in non-dimensional form, suitable for simulation of turbulent compressible flow with the LES approach. The filtered equations in compact form read

$$
\partial_{t} \mathbf{U}+\nabla \cdot \mathbf{F}(\mathbf{U})=0
$$

where $\mathbf{U}=\left[\bar{\rho}, \bar{\rho} \widetilde{\mathbf{u}}^{T}, \bar{\rho} \widetilde{e}\right]^{T}$ are the prognostic filtered variables; namely, density $\bar{\rho}$, momentum $\bar{\rho} \widetilde{\mathbf{u}}$ and total energy per unit volume $\bar{\rho} \widetilde{e}=\bar{\rho}\left(\widetilde{u}_{i} \widetilde{u}_{i} / 2+\widetilde{e}_{i}\right)$, sum of kinetic energy and internal energy $\bar{\rho} \widetilde{e}_{i}$. Here $\checkmark$ represents the space filter operator usually considered in LES, while the Favre filter operator $\widetilde{\sim}=\bar{\rho} / \bar{\rho}$ is introduced as usual to avoid additional subgrid terms in the mass conservation equation. In equation 
(1) $\mathbf{F}=\mathbf{F}^{\mathrm{c}}-\mathbf{F}^{\mathrm{v}}-\mathbf{F}^{\mathrm{sgs}}$ is composed by the convective $\mathbf{F}^{\mathrm{c}}$, the viscous $\mathbf{F}^{\mathrm{v}}$ and the subgrid $\mathbf{F}^{\text {sgs }}$ fluxes expressed as

$$
\mathbf{F}^{\mathrm{c}}=\left[\begin{array}{c}
\bar{\rho} \widetilde{\mathbf{u}} \\
\bar{\rho} \widetilde{\mathbf{u}} \otimes \widetilde{\mathbf{u}}+\frac{1}{\gamma M a^{2}} \bar{p} \mathbb{I} \\
(\bar{\rho} \widetilde{e}+\bar{p}) \widetilde{\mathbf{u}}
\end{array}\right], \quad \mathbf{F}^{\mathrm{v}}=\left[\begin{array}{c}
0 \\
\frac{1}{R e} \widetilde{\sigma} \\
\frac{\gamma M a^{2}}{R e} \widetilde{\mathbf{u}}^{T} \widetilde{\sigma}-\frac{1}{k \operatorname{RePr}} \widetilde{\mathbf{q}}
\end{array}\right], \quad \mathbf{F}^{\mathrm{sgs}}=\left[\begin{array}{c}
0 \\
\tau \\
\frac{1}{k} \mathbf{Q}+\frac{\gamma M a^{2}}{2}\left(J-\tau_{k k} \widetilde{\mathbf{u}}\right)
\end{array}\right]
$$

where the Mach number $M a$, the Reynolds number $R e$, the Prandtl number $P r$, the specific heat ratio $\gamma=c_{p} / c_{v}$ and $k=c_{p} / R$ appear, being $R$ the ideal gas constant. The viscous stresses $\sigma$ and the heat flux $\mathbf{q}$ are defined by the constitutive relations

$$
\sigma_{i j}=2 \mu\left(\widetilde{\mathcal{S}}_{i j}-\frac{1}{3} \widetilde{\mathcal{S}}_{k k} \delta_{i j}\right), \quad q_{i}=\mu \partial_{i} \widetilde{T}
$$

where $\widetilde{S}_{i j}=\left(\partial_{i} \widetilde{u}_{j}+\partial_{j} \widetilde{u}_{i}\right) / 2$ is the strain rate tensor and the dynamic viscosity depends on the temperature according to the Sutherland's law $\mu=\widetilde{T}^{0.7}$. Finally the system is closed with the equation of state for ideal gas $\bar{p}=\bar{\rho} \widetilde{T}$ relating the pressure $\bar{p}$ to the temperature $\widetilde{T}$.

The subgrid flux introduced in equation (2) contains the turbulent stresses $\tau_{i j}=\bar{\rho} u_{i} u_{j}-\bar{\rho} \widetilde{u}_{i} \widetilde{u}_{j}$, the subgrid heat flux $\mathbf{Q}=\overline{\rho T \mathbf{u}}-\bar{\rho} \widetilde{T} \widetilde{\mathbf{u}}$ and the turbulent diffusion flux $\mathbf{J}=\overline{\rho \mathbf{u} u_{k} u_{k}}-\bar{\rho} \widetilde{\mathbf{u}} \widetilde{u}_{k} \widetilde{u}_{k}$ which are modelled using the anisotropic model proposed in [1], extended to compressible flows in [2, 3] and briefly recalled in the following. In this model the subgrid stress tensor $\tau$ is assumed to be proportional to the strain rate tensor $\widetilde{S}$ through a fourth order
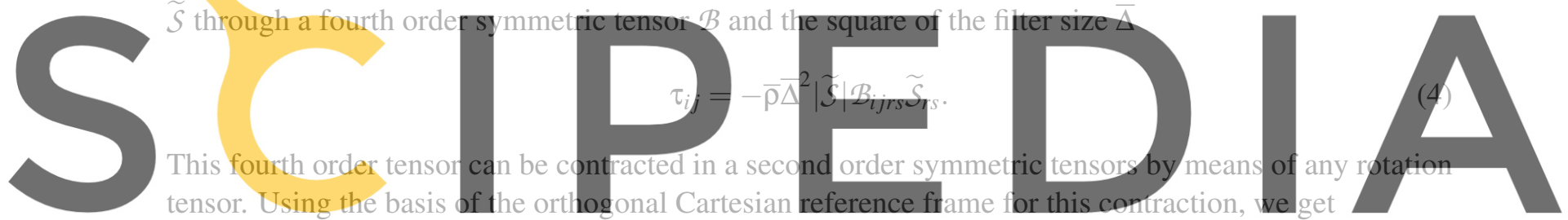

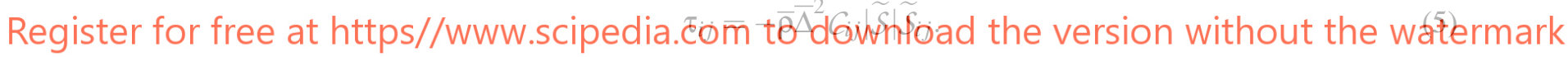

Then the six independent element of the tensor $C$ are determined applying the Germano's dynamic procedure. The same anisotropic dynamic procedure is applied to determine the subgrid heat flux and the subgrid diffusion flux which read

$$
Q_{i}=-\bar{\rho} \bar{\Delta}^{2} C_{i}^{Q}|\widetilde{\mathcal{S}}| \partial_{i} \widetilde{T}, \quad J_{i}=-\bar{\rho} \bar{\Delta}^{2}|\widetilde{\mathcal{S}}| C_{i}^{J} \partial_{i}\left(\frac{1}{2} \widetilde{u}_{k} \widetilde{u}_{k}\right)
$$

\subsection{The numerical model}

The equations are discretized in space by a finite element approach based on the Local Discontinuous Galerkin (LDG) [8] approach. In the LDG method the auxiliary variable $\boldsymbol{G}$ is defined for which an additional equation is introduced. In this way the previous equations are reduced to the first order system

$$
\begin{aligned}
\partial_{t} \mathbf{U}+\nabla \cdot \mathbf{F}(\mathbf{U}, \boldsymbol{G}) & =0 \\
\boldsymbol{G}-\nabla \boldsymbol{\varphi} & =0
\end{aligned}
$$


in which $\varphi=\left[\widetilde{\mathbf{u}}^{T}, \widetilde{T}\right]^{T}$. The weak form of the equations is discretized on the discontinuous finite element space defined over a tessellation of non-overlapping tetrahedral elements. The Rusanov form for the convective flux is used in the present work, while centred fluxes are applied to viscous, subgrid and gradient fluxes [13]. A modal DG formulation is applied which means that the hierarchical orthonormal basis obtained from the Legendre polynomials are used for both the discontinuous finite element subspace and the prognostic unknowns, while symmetric quadrature rules [28] are applied to compute the projection in the physical space. Since the used formulae must be exact at least up to twice the polynomial degree $q_{K}$ in each element $K$, the integrals on internal boundaries between two elements are computed using the maximum degree between the two elements.

As presented in [25], the grid filter - is equivalent to the projection over the solution subspace so that the filtered prognostic quantities can be identified with their numerical solution counterparts. Moreover the discretised filtered equations are advanced in time with the explicit, five stages fourth order, Strong Stability Preserving Runge-Kutta method proposed in [21].

The illustrated numerical model is implemented in a numerical code based on EEMilaro [12], a generic finite element library written using latest Fortran and MPI standards. A more detailed description of the numerical code can be found in [2].

\subsection{The polynomial adaptivity indicator}

In each element the maximum polynomial degree is selected on the base of an indicator which should

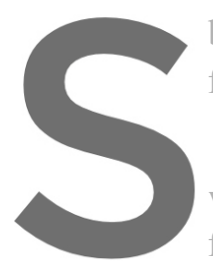

be sensitive to the local co

function

where $<\cdot>$ represents the expected value operato

for each couple of elemen
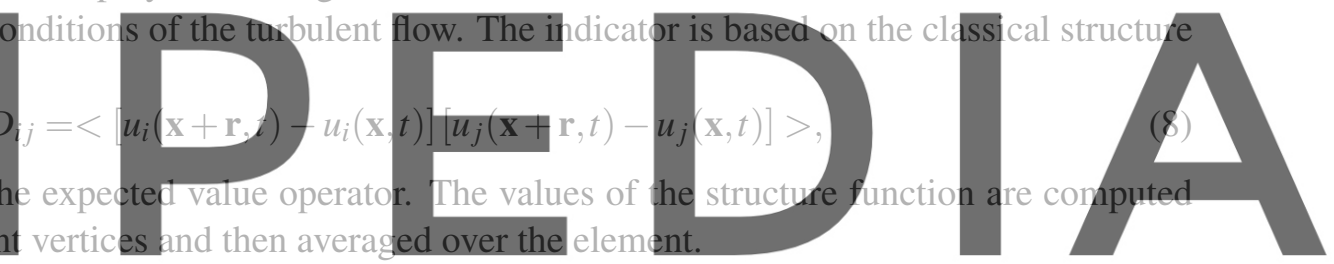

The structure function estimates the lack of correlation in the velocity values at the two points $\mathbf{x}$ and

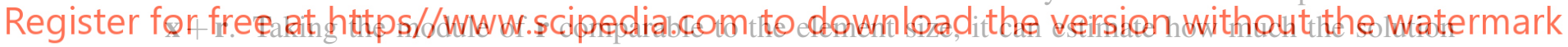

is fluctuating inside each element. Large values of the structure function will denote the requirement

for more resolution while small values will indicate laminar conditions or a very well resolved turbulent

region such that the reduction of the resolution in that element is possible.

Since in homogeneous isotropic turbulence the subgrid scale model takes charge of the turbulent dissipation, the contribution due to homogeneous isotropic turbulence

$$
D_{i j}^{i s o}(\mathbf{r}, t)=D_{N N}(r, t) \delta_{i j}+\left(D_{L L}(r, t)-D_{N N}(r, t)\right) \frac{r_{i} r_{j}}{r^{2}}
$$

is removed from the structure function (8). $D_{L L}, D_{N N}$ are the longitudinal and transverse structure functions, respectively, determined by a least square fit of equation (9) to the structure function values within the element. The polynomial adaptivity indicator is then defined as:

$$
\operatorname{Ind}_{S F}(K)=\sqrt{\sum_{i j}\left[D_{i j}(K)-D_{i j}(K)^{i s o}\right]^{2}} .
$$



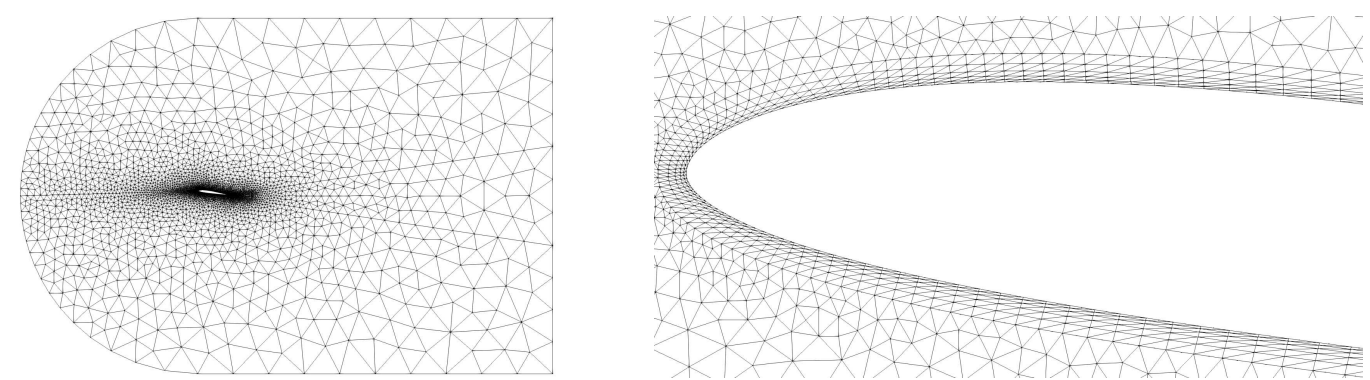

Figure 1: The two dimensional sketch of the computational mesh: the global view (left) and the detail around the airfoil (right)

This indicator well quantifies the turbulence intensity suggesting where the turbulent flow features allow for a coarser resolution and more modelling. Although the structure function indicator (10) is more complex than other indicator based on the numerical error, it could give more physical insight into the flow conditions. Note that an indicator based on the interpolated values of the solution at different points of the element was already presented in [7], but in that case, the values were used to evaluate a local approximation of the velocity gradients.

In the present simulations, the maximum polynomial degrees range between 2 and 4 . Two indicator thresholds $\varepsilon_{1}<\varepsilon_{2}$ has been introduced. The maximum polynomial degree 2 is assigned in the elements with a value of the indicator lower than a threshold $\varepsilon_{1}$, while the degree is limited to 4 in the cells with

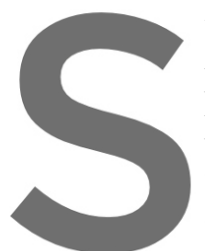
values higher than the procedure, the degree p-adaptive procedure can a parallel run. In the $n$ at the beginning of the most efficient configuration, the polynomial adaptivit
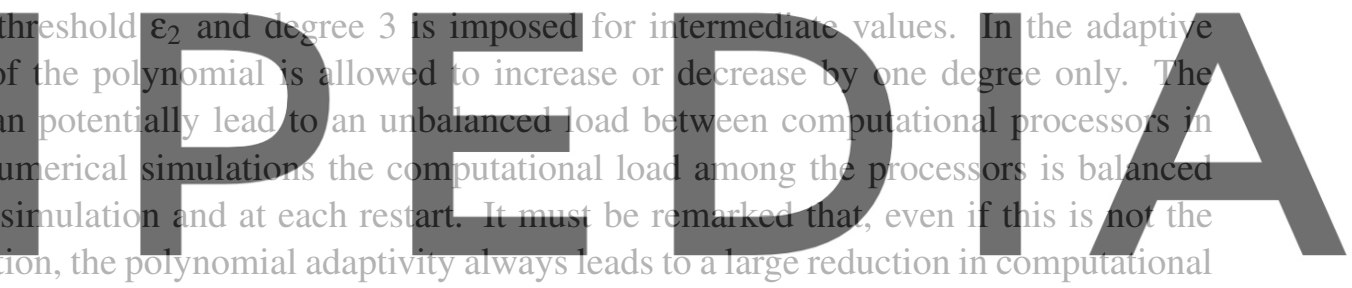
effort. Further work on code optimisation is needed in order to implement an efficient load balancing

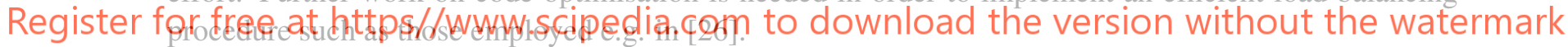

\section{NUMERICAL SIMULATIONS}

The p-adaptive solver is first applied to the simulation of the transitional flow over the SD7003 infinite wing. Then a vortex is introduced in the field developed around the airfoil to simulate the BVI phenomenon. Simulations are carried out at a free-stream Mach number $M a=0.2$ and a Reynolds number $R e=60000$. All the lengths are normalised by the chord. The angle of attack $\alpha=8^{\circ}$ is prescribed. The flow in these conditions is characterised by a short laminar separation bubble close to the LE with a turbulent reattachment. The airfoil geometry is extruded 0.2 in the spanwise direction, as in [5]. The LE is located in the origin of the cartesian reference frame. The inflow, the upper and lower boundaries are distant 5 unit from the LE while the outflow boundary is located at 10 downstream the airfoil. The non-slip, isothermal Dirichlet boundary condition with $T_{w}=1$ are imposed at the surface of the airfoil. The inflow condition corresponds to far-field values $U_{\infty}=(1,0,0)$ and $T_{\infty}=1$. Sponge layers have been applied on the outflow, on the upper and on the lower boundary to avoid reflections [9]. 


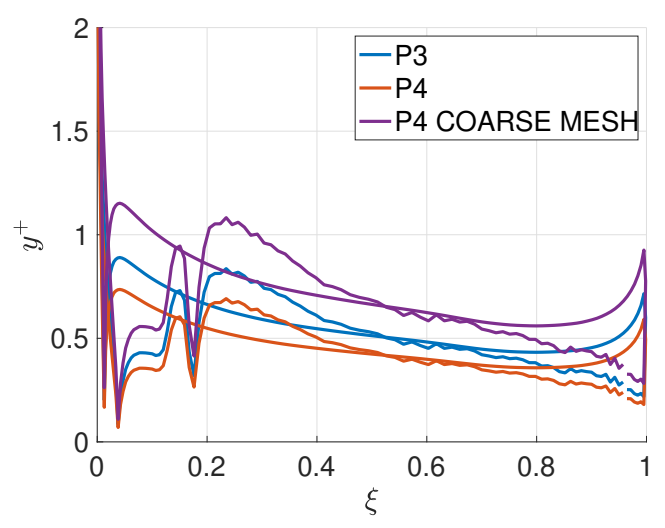

Figure 2: Distance from the wall of the first point in wall unit for different resolutions.

A two-dimensional sketch of the grid is represented in Figure 1 where a refinement line upstream of the airfoil, to prevent the over dissipation of the vortex, is visible. A structured grid has been constructed inside a 0.01 thick layer around the airfoil. This layer is divided into $N_{n}$ and $N_{z}$ layers in normal to the wall and spanwise directions respectively. Each hexahedron of these layers is divided in 6 tetrahedra. Being $l_{i}$ the size of the hexahedra in the $i$ direction, the equivalent resolution in space can be determined as
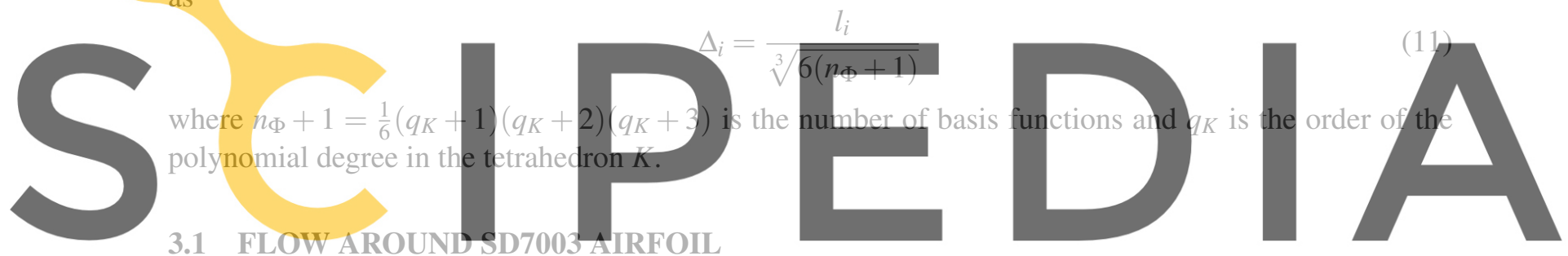

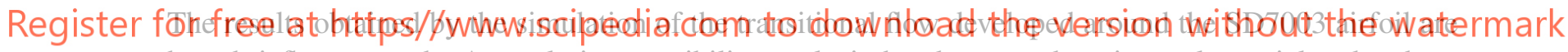
here briefly resumed. A resolution sensibility analysis has been made using polynomial order three and four with two different grids which details are reported in Table 1. A sketch of the finer mesh is represented in Figure 1.

Table 1: Details of the two adopted meshes

\begin{tabular}{|c|c|c|c|c|c|}
\hline Mesh & N. Elements & $\Delta_{1^{\mathrm{st}, n}} / c$ & $N_{n}$ layers & $\Delta_{z} / c$ & $N_{z}$ layers \\
\hline Coarse & 57479 & $7.45 \times 10^{-4}$ & 4 & $1.83 \times 10^{-2}$ & 6 \\
\hline Fine & 110577 & $4.76 \times 10^{-4}$ & 5 & $1.38 \times 10^{-2}$ & 8 \\
\hline
\end{tabular}

An estimate of distance from the wall of the first point in wall coordinates for uniform polynomial degree is shown in Figure 2 where $\xi$ indicates the coordinate parallel to the chord. It has been computed using the friction coefficient distribution of the $\mathrm{P} 4$ simulation. Concerning the $\mathrm{P}$-adaptive simulation, since the polynomial degree in the boundary layer is 3 or 4 , the distance from the wall is always between the P4 and the P3 curves. The first point height in wall-units is below one for the fine mesh, as required for an 

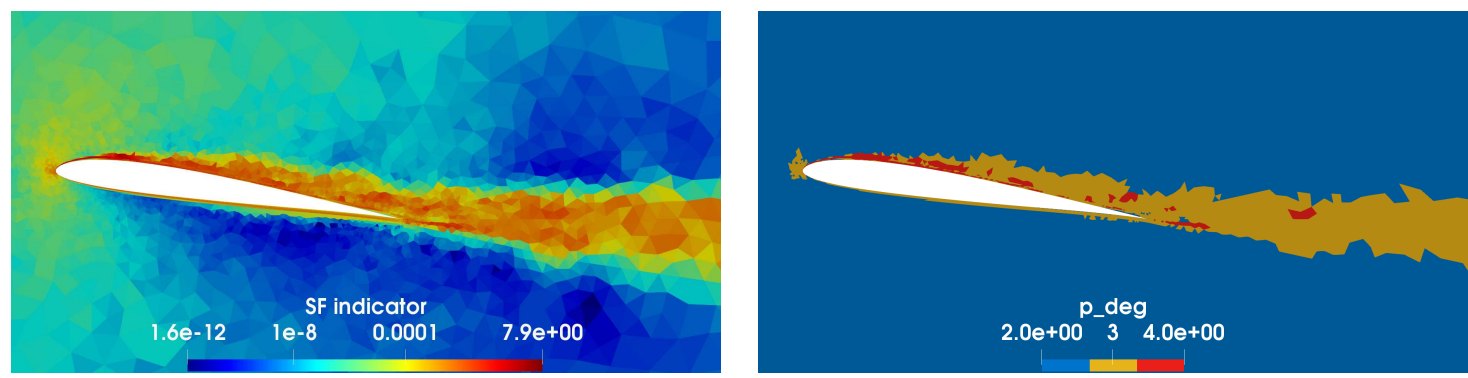

Figure 3: Value of the SF indicator in the plane $z=0.2$, in logarithmic scale (left); polynomial degree distribution obtained with the thresholds $\varepsilon_{1}=10^{-4}$ and $\varepsilon_{2}=10^{-2}$ (right).

adequate resolution of the boundary layer, with the exception of a small region near the stagnation point where the friction coefficient tends to infinity. The polynomial degree distribution has been recovered with the static p-adaptive approach: the value for the SF indicator has been computed from an underresolved $\mathrm{P} 2$ simulation and averaged over 0.5 time units. A $2 \mathrm{D}$ view of the resulting value of the indicator in the domain and the resulting polynomial degree distribution are shown in Figure 3. The indicator is able to capture the turbulent wake region as well as the boundary layer on the pressure side. The 4th order polynomial is also used in the laminar boundary layer and in the transitional region on the suction side. A very similar polynomial distribution obtained with a spectral decay smoothness indicator on the SD7003 airfoil is presented in [26].

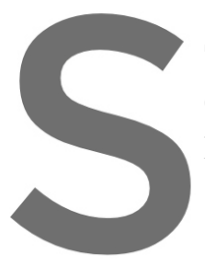

Table 2: Comparison betwed drag and the moment coefficic positions of the separation, o
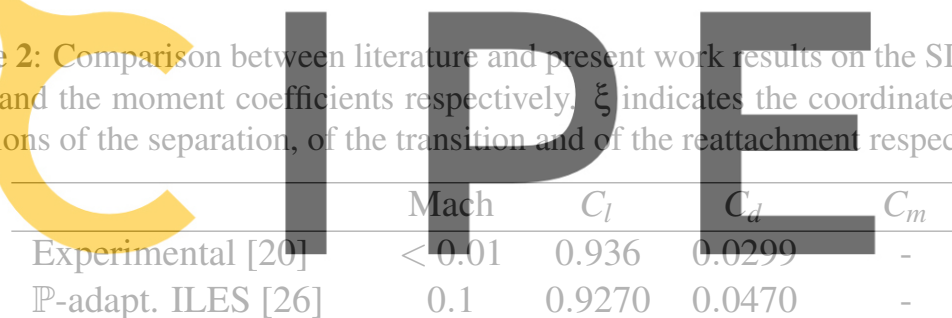

0.9270

0.0470

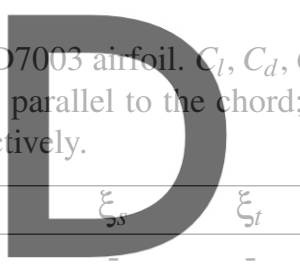

0.0301

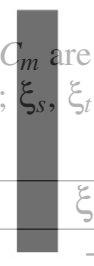

0.3123

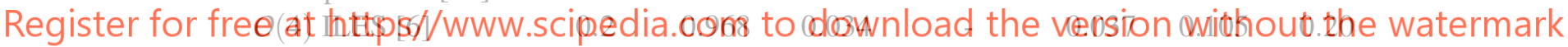

\begin{tabular}{llllllll}
$\mathbb{P}^{4}$ LES present & 0.2 & 0.9738 & 0.0356 & -0.0192 & 0.038 & 0.1 & 0.173 \\
$\mathbb{P}$-adapt. LES present & 0.2 & 0.9693 & 0.0346 & -0.0186 & 0.038 & 0.1 & 0.175 \\
\hline
\end{tabular}

The main results obtained with the fine grid are reported in Table 2. They have been averaged for at least 2 convective time units after the statistical convergence has been verified. In particular, very good agreement is recovered comparing the present results with the ILES of [6] performed at the same Mach number 0.2. The influence of the Mach number on the pressure distribution and on the separation bubble should be further investigated in a future work. Considering the $\mathbb{P}^{4}$ solution on the fine mesh as a reference, it can be observed that the drag and moment coefficients computed with the polynomial adaptivity differs of $3 \%$ while the differences for all the others quantities are negligible. 


\subsection{SIMULATION OF PARALLEL BVI}

In the present work the parallel interaction between a vortex and an SD7003 airfoil at $\alpha=8^{\circ}$, Reynolds number equal 60000 and Mach number 0.2 is investigated. The type of interaction studied in the present work is usually called viscous interaction because of the impact of the vortex viscous core on the leading edge (LE). As a result, the primary vortex splits into two secondary vortices which are advected downstream and, eventually, break down into smaller scales structures [27]. As far as the authors' knowledge, there are not numerical simulations or experimental measurements in literature which can be used for a direct comparison. However some examples of similar works are presented in [11]. Experimental studies on parallel BVI on the SD7003 airfoil have been made by [18] but they cannot be used for comparison, not only for the different Reynolds number, but, mostly, because two vortices are present in that experimental set-up.

A vortex, with axis parallel to the wing and rotating in the counterclockwise direction, has been introduced in the developed velocity field obtained by the simulation of the flow around the SD7003 airfoil described in the previous subsection, in order to simulate the BVI phenomenon. The definition of the vortex is the one used by $[15,24]$ inducing the velocity and pressure

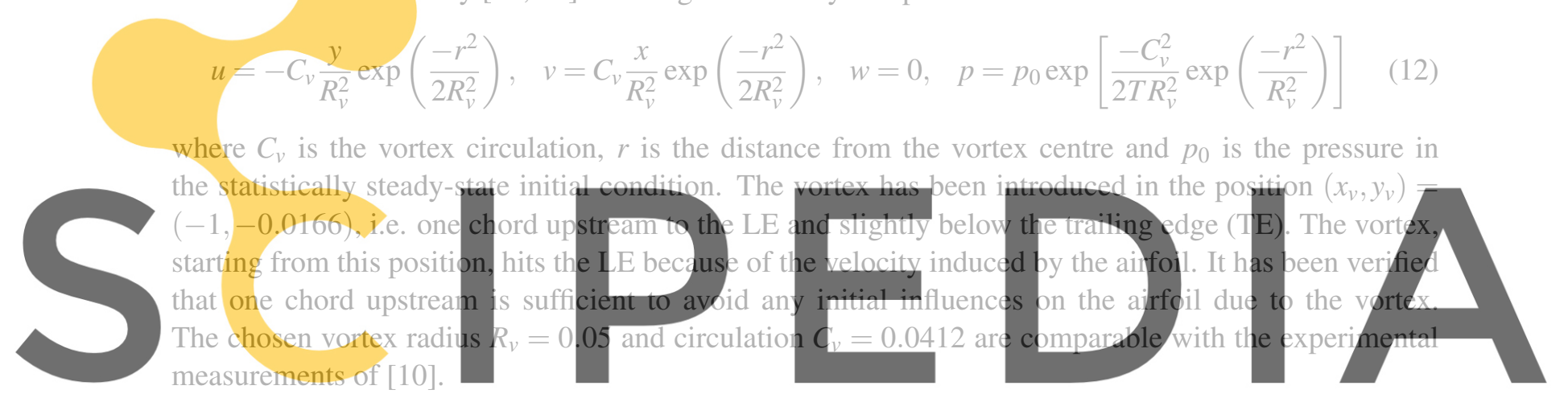

The dynamic p-adaption has been applied with the same thresholds used for the static adaptivity in

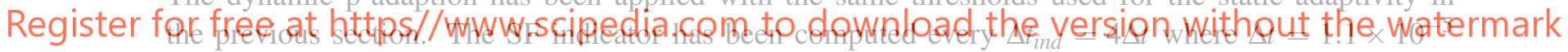

is the computational time step. Then it was averaged over $\Delta t_{\text {adapt }}=30 \Delta t_{\text {ind }}$ before the polynomial distribution should be updated. This procedure ensure to accurately follow the vortex advected with a velocity comparable to the undisturbed one. Furthermore, the unsteady flow above the airfoil is correctly represented since the adaption interval $\Delta t_{\text {adapt }}$ is also much lower than the vortex shedding period on the separation bubble. Figure 4 shows the resulting polynomial degree distribution for different time instants. It is evident how the SF indicator is able to detect and follow the advected vortex during time. After the impact of the primary vortex on the LE of the airfoil the SF indicator detects accurately the position of the two secondary vortices.

From an engineering point of view, one of the most interesting results is the amplitude in the oscillations of the loads for structural analysis. The force coefficients are compared in Figure 5 with the reference $\mathbb{P}$-adaptive simulation without the vortex. The force coefficients do not deviate from the reference simulation until $t \approx 0.6$ when the vortex is 0.4 upstream the LE. The drag is the first component of the aerodynamic force to change. It decreases and reaches the minimum value of $C_{d, \min }=-0.014$ at $t=1.04$ when the low pressure core of the vortex acts on the LE. This is confirmed by the fact that the stronger variation in the drag is pressure-driven. On the other hand, the lift increases because the vortex is ro- 


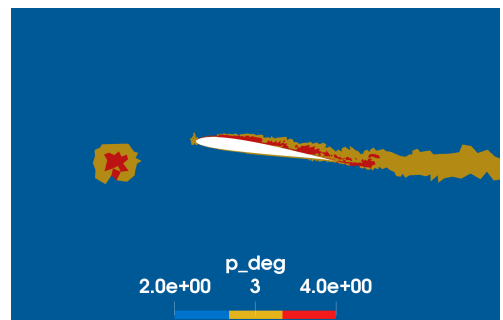

(a) $t=0.5$

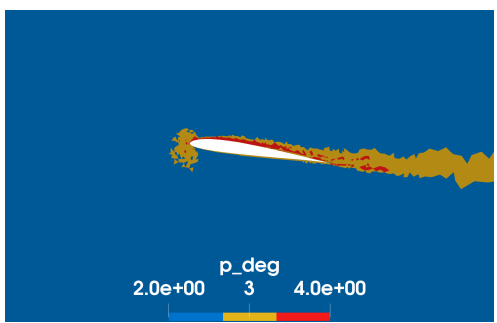

(b) $t=1.1$

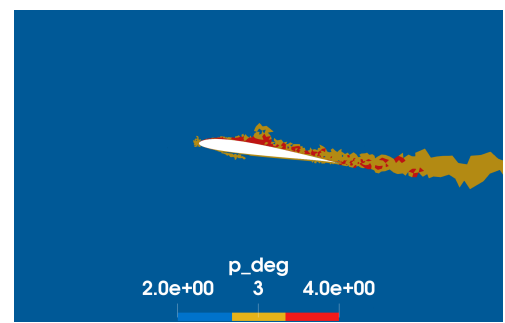

(c) $t=1.4$

Figure 4: $2 \mathrm{D}$ view in the plane $z=0.2$ of the polynomial degree distribution at different times $t$.

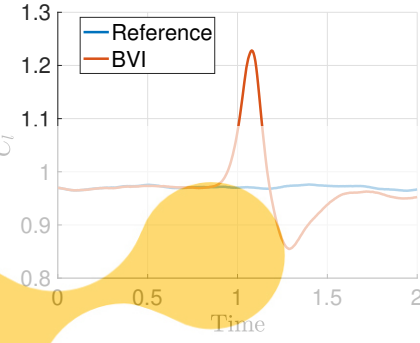

(a) Lift coefficient

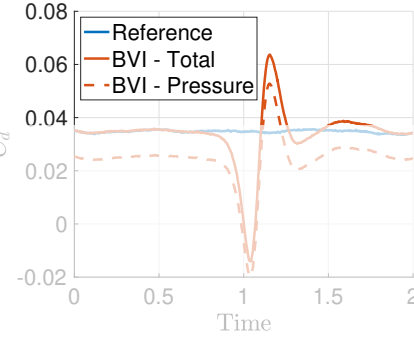

(b) Drag coefficient

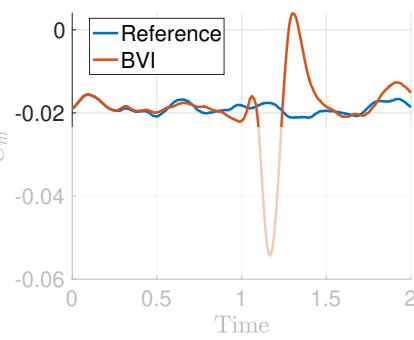

(c) Moment coefficient

Figure 5: Forces coefficients in function of time during the BVI, compared with the reference simulation without

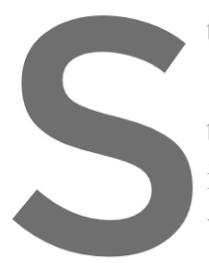
the vortex.

tating counter-clockwis

its maximum value $C_{l, n}$

value. The minimum value
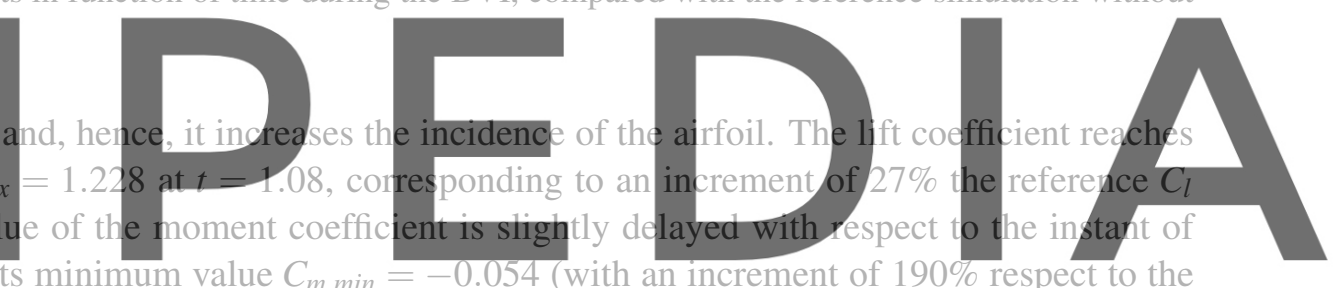

maximum lift, reaching its minimum value $C_{\text {m.min }}=-0.054$ (with an increment of $190 \%$ respect to the

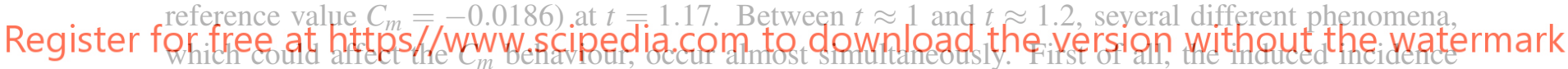

changes the pressure distribution mainly for $0<\xi<0.2$ in both the pressure and suction sides of the airfoil, as showed in Figure 6 for $t=1.0$, resulting in an increase of the lift coefficient. After have hit the LE at $t=1.04$ (corresponding to the minimum drag), the vortex splits in two: the low-pressure core causes the minimum clearly visible on the pressure side in the curve for $t=1.1$, while the secondary vortex has not yet started being advected on the suction side and, hence, the pressure is still higher than its reference value. After the secondary vortex is moved on the pressure side, the pressure results significantly higher than in the reference condition, especially for $0<\xi<0.3$ (curve for $t=1.2$ in Figure 6) and consequently the moment coefficient reaches its minimum value. This effect is accentuated, on the pressure-side, by the low-pressure core of the secondary vortex at $\xi \approx 0.1$ and the higher pressure for $\xi>0.5$ caused by the acoustic wave generated by the impact of the vortex on the LE, as shown in Figure 7 (b). The maximum value of the drag coefficient $C_{d, \max }=0.064$ is reached at $t=1.15$, representing an increase of $85 \%$ with respect to the reference value. After the positive peak, the lift decreases reaching the value $C_{l, \min }=0.855$, with a defect of $18 \%$ respect to the reference value, at the time $t=1.3$ when the moment coefficient reaches the maximum $C_{m, \max }=0.004$. This is probably due to a small separation induced by the secondary vortex on the suction-side. It is worth noting that at $t=1.9$ the drag coefficient 


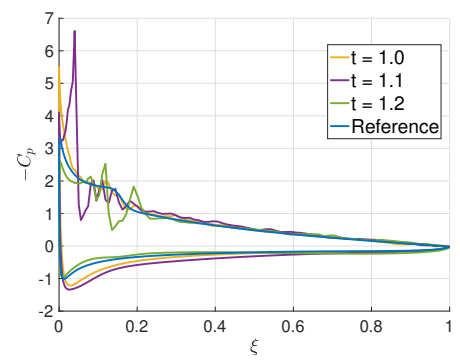

Figure 6: Pressure coefficient $C_{p}$ at different times during the BVI, compared with the reference simulation without the vortex.

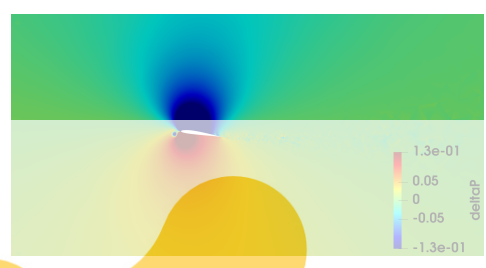

(a) $t=0.9$

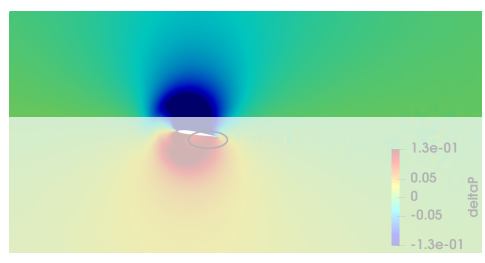

(b) $t=1.2$

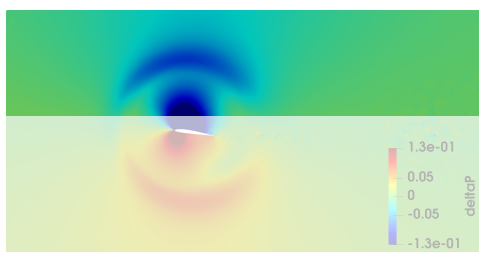

(c) $t=1.4$

Figure 7: Visualization of the pressure perturbation $p-p_{r}$ in the plane $z=0.2$ at different time $t$. The circle highlight the high pressure caused by the acoustic wave generated by the impact of the vortex on the LE.

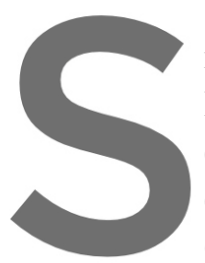

reaches the steady-state val
higher than the correspond
end of the transient. This p
circulating region on the suctictices during
of the small vortice
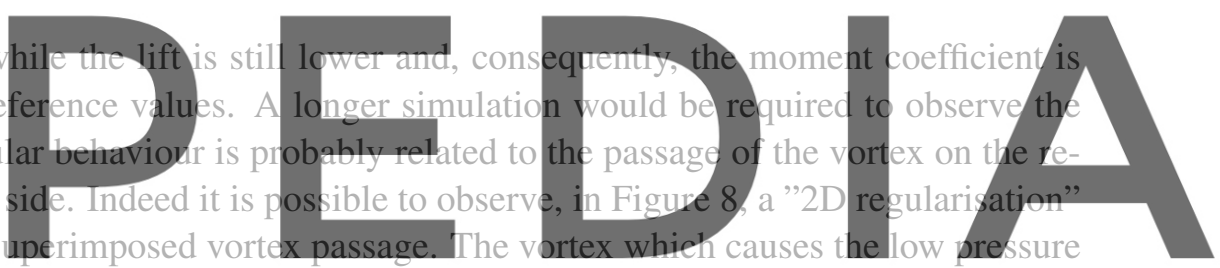

peak in the at $t=1.1$ is clearly visible in Figure 8(b). After the passage of the vortex (Figure 8(c)) it is

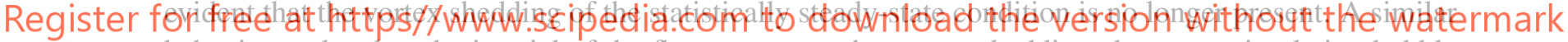
behaviour related to the inertial of the flow to restore the vortex shedding above a recirculating bubble after the BVI, has been found also in a different configuration [4]. The highlighted delay of the lift and the moment to recover the steady values is an important result because it can strongly affect the airfoil global performances.

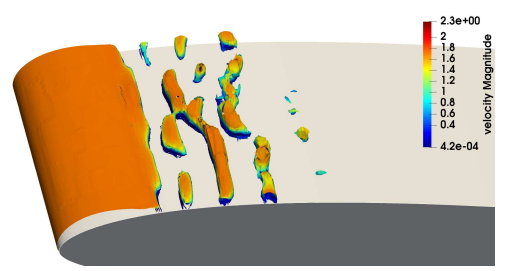

(a) $t=0.5$

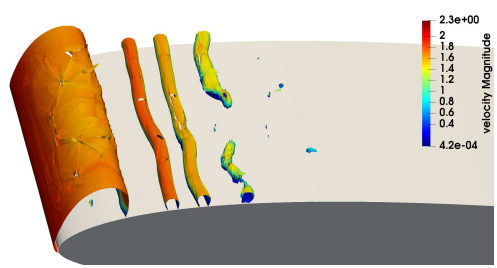

(b) $t=1.1$

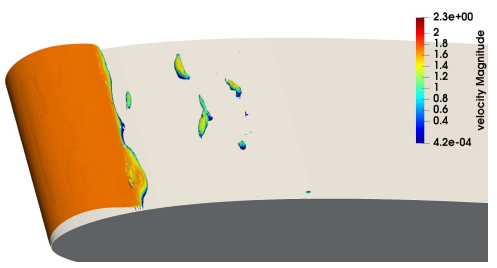

(c) $t=1.6$

Figure 8: Pressure iso-surfaces in the region of the recirculating bubble on the suction side. 


\section{CONCLUSIONS}

The parallel, viscous BVI has been studied using the dynamic P-adaptive approach in an LDG framework. The results are compared with the statistically steady flow around the airfoil, without vortex, to investigate the effects of the interaction on the aerodynamic forces. Lift, drag and moment coefficients are all strongly affected by this interaction. This effect is ascribed to many different factors: the induced variation of incidence causes the positive peak of the lift; the pressure coefficient distribution change because the passage of the secondary vortices cores and of the acoustic wave generated by the vortex impact on the LE; the parallel vortex inhibit the vortex shedding above the recirculating region. It has been noticed that the transient of the lift and moment coefficients is longer than the one of the drag which rapidly comes back to the statistically steady-state condition. This has been explained with the less intense vortex shedding phenomenon above the separation bubble which persists over time after the BVI.

\section{ACKNOWLEDGEMENTS}

We acknowledge the CINECA award under the ISCRA initiative, for the availability of high performance computing resources and support.

\section{REFERENCES}

[1] Abbà, A., Cercignani, C. and Valdettaro, L. Analysis of Subgrid Scale Models. Comput. \& Math. Appl. (2003) 46:521-535.

[2] Abbà, A., Bonaventura, L., Nini, M. and Restelli, M. Dynamic models for Large Eddy Simulation of compressible flows with a high order DG method. Comput. \& Fluids (2015) 122:209-222.

[3] Abbà, A., Campaniello, D. and Nini, M. Filter size definition in anisotropic subgrid models for large eddy simulation on irregular grids. J. of Turb. (2017)18(6):589-610.

[4] Abba', A., Recanati, A., Tugnoli, M. and Bonaventura, L. Dynamical p-adaptivity for LES of compressible flows in a high order DG framework. J. Comp. Phys. (2020) 420:109720.

[5] Bassi, F., Botti, L., Colombo, A., Crivellini, A., Ghidoni, A. and Massa, F. On the development of an implicit high-order discontinuous galerkin method for DNS and implicit LES of turbulent flows. Europ. J. Mech. - B/Fluids (2016)55:367-379

[6] Boom, P., and Zingg, D. Time-accurate flow simulations using an efficient newton-krylov-schur approach with high-order temporal and spatial discretization. 51st AIAA Aerospace Sciences Meeting(2013).

[7] Burbeau, A. and Sagaut, P. A dynamic $p$-adaptive discontinuous Galerkin method for viscous flow with shocks. Comput. \& Fluids (2005) 34:401-2417.

[8] Cockburn, B. and Shu, C.W. The Runge-Kutta Discontinuous Galerkin Method for Conservation Laws. J. Comp. Phys. (1998) 141:198-224.

[9] Crivellini, A. Assessment of a sponge layer as a non-reflective boundary treatment with highly accurate gust-airfoil interaction results. Int. J Comput. Fluid Dyn. (2016) 30(2):176-200. 
[10] Droandi, G., Gibertini, G. and Zanotti, A. Perpendicular blade-vortex-interaction over an oscillating airfoil in light dynamic stall. J. Fluids and Struct. (2016) 65:472-494.

[11] Felten, F. and Lund, T. Numerical Simulation of Parallel Airfoil/Vortex Interaction Using a Zonal Hybrid RANS/LES Method. 17th AIAA Computational Fluid Dynamics Conference (2005).

[12] FEMilaro, a finite element toolbox. Available under GNU GPL v3 https://bitbucket.org/mrestelli/femilaro/wiki/Home

[13] Giraldo, F.X. and Restelli,M. A study of spectral element and discontinuous Galerkin methods for the Navier-Stokes equations in nonhydrostatic mesoscale atmospheric modeling: equation sets and test cases. J. Comp. Phys. (2008) 227:3849-3877.

[14] Ilie, M. A fully-coupled CFD/CSD computational approach for aeroelastic studies of helicopter blade-vortex interaction. Appl. Math. Comput. (2019) 347:122-142.

[15] Lodato, G., Domingo, P. and Vervisch, L. journal = JCP,Three-dimensional boundary conditions for direct and large-eddy simulation of compressible viscous flows. J. Comp. Phys. (2008) 227:51055143.

[16] Morvant, R., Badcock, K.K.J. and Barakos, G.G.N. Aerofoil-Vortex Interaction Using the Compressible Vorticity Confinement Method. AIAA J (2005) 43(1):63-75.

[17] Peng, D. and Gregory, J.W. Vortex dynamics during blade-vortex interactions. Phys. Fluids (2015) 27(5):053104.

[18] Rival, D., Manejev, R. and Tropea, C. Measurement of parallel blade-vortex interaction at low Reynolds numbers. Exp. in Fluids (2010) 49:89-99.

[19] Rockwell, D. Vortex-body interactions. Ann. Rev. Fluid Mech. (1988) 30(1):199-229

[20] Broeren, A.P., Giguere, P., Selig, M.S. and Guglielmo, J.J. Summary of Low-Speed Airfoil Data. SoarTech Publications Vol. I., (1995).

[21] Spiteri, R.J., and Ruuth, S.J. A New Class of Optimal High-Order Strong-Stability-Preserving Time Discretization Methods. SIAM J. Num. Anal. (2002) 40:469-491

[22] Tanabe, Y., Saito, S., Takasaki, K. and Fujita, H. A parametric study of parallel blade-vortexinteraction noise. Noise Control Eng. J. (2009) 57(5):420-433.

[23] Tugnoli, M., Abbà, A., Bonaventura, L. and Restelli, M. A locally p-adaptive approach for Large Eddy Simulation of compressible flows in a DG framework. J. Comp. Phys. (2017) 349:33-58.

[24] Tugnoli, M., Abbà, A., Bonaventura, L. Dynamical degree adaptivity for DG-LES models. Proceedings of the ICOSAHOM 2018 Conference (2019).

[25] van der Bos, F., van der Vegt, J.J.W., and Geurts, B.J. A Multi-Scale Formulation for Compressible Turbulent Flows Suitable for General Variational Discretization Techniques. Comput. Methods Appl. Mech. Engng. (2007) 196:2863-2875.

[26] Wang, K., Gobbert, M.K. and Yu, M. A dynamically load-balanced parallel $p$-adaptive implicit high-order flux reconstruction method for under-resolved turbulence simulation. J. Comp. Phys. (2020) 417:109581. 
[27] Wilder, M.C. and Telionis, D.P. Parallel blade-vortex interaction. J. Fluids and Struct. (1988) 12(7):801-838.

[28] Zhang, L., Cui, T. and Liu, H. A set of symmetric quadrature rules on triangles and tetrahedra. J. Comp. Math. (2009) 27(1):89-96. 